\title{
Potentially Inappropriate Medications in Elderly Haemodialysis Patients Using the STOPP Criteria
}

\author{
Krystina Parker $^{1,2}$ (1) - Willy Aasebø ${ }^{1} \cdot$ Knut Stavem $^{2,3,4}$
}

Published online: 8 August 2016

(c) The Author(s) 2016. This article is published with open access at Springerlink.com

\begin{abstract}
Background Polypharmacy is commonly applied to elderly haemodialysis patients for treating terminal renal failure and multiple co-morbidities. Potentially inappropriate medications (PIMs) in multidrug regimens in geriatric populations can be identified using specially designed screening tools.

Objective The aims of this study were to estimate the prevalence of PIMs by applying the Screening Tool of Older Persons' Prescriptions (STOPP) criteria and the Beers criteria to elderly haemodialysis patients and to assess the association of some risk factors with the presence of PIMs.

Methods Fifty-one elderly haemodialysis patients participated; their median age was 74 (range 65-89) years, and $77 \%$ of them were male. Demographic data, co-morbidity and medication lists were collected from the electronic medical records of the patients. The STOPP criteria were applied by two physicians independently to identify PIMs. The association of some risk factors with PIMs were assessed using Fisher's exact test.

Results The patients used a median of 13 (range 7-21) medications per day. The overall prevalence of PIMs using
\end{abstract}

Krystina Parker

krystina.parker@medisin.uio.no

1 Medical Division, Department of Nephrology, Akershus University Hospital, 1478 Lørenskog, Norway

2 Institute of Clinical Medicine, University of Oslo, Oslo, Norway

3 Medical Division, Department of Pulmonary Medicine, Akershus University Hospital, Lørenskog, Norway

4 HØKH, Department of Health Services Research, Akershus University Hospital, Lørenskog, Norway the STOPP criteria was $63 \%$, and using the Beers criteria was $43 \%$. The most prevalent PIMs were proton-pump inhibitors. Benzodiazepines and first-generation antihistamines were related to side effects such as falls in the previous 3 months, and calcium-channel blockers were associated with chronic constipation. The number of PIMs was not significantly associated with number of medications, age, sex and co-morbidity.

Conclusions The STOPP criteria revealed a high prevalence of PIMs in a population of elderly patients receiving haemodialysis.

\section{Key Points}

Potentially inappropriate medications according to STOPP criteria were identified in $63 \%$ of elderly haemodialysis patients and in $43 \%$ using the Beers criteria.

Proton pump inhibitors were the most prevalent drugs according to STOPP criteria.

The number of potentially inappropriate medications was not significantly associated with number of medications, age, sex and co-morbidity.

\section{Background}

Elderly haemodialysis patients are considered to be a vulnerable group due to the presence of renal failure with underlying co-morbidities that require the use of multiple 
drugs. Drug efficacy in these patients is influenced by agerelated changes, altered nutritional state and the haemodialysis treatment they receive. Haemodialysis alters the pharmacokinetics and pharmacodynamics of many drugs due to the presence of continuous changes in the fluid balance and uraemic toxin levels, and this may increase the risk of drug-to-drug interactions and adverse side effects in haemodialysis patients.

International guidelines for management of patients with renal failure point to the importance of dose reduction or discontinuation of certain drugs and provide cautionary notes for prescribing (Kidney Disease Improving Global Outcome [KDIGO] 2013) [1] or recommend regular review of medication lists (Kidney Disease Outcome Quality Initiative [KDOQI]) [2]. Several other approaches can be used to identify potentially inappropriate medications (PIMs), such as a targeted multidisciplinary team approach, consulting with pharmacists [3-5] or using validated screening tools [6-8]. Two recent studies used such a screening tool called the Beers criteria $[9,10]$, reporting a high prevalence of PIMs in elderly patients with chronic kidney disease and end-stage renal disease (ESRD). The Screening Tool of Older Persons' Prescriptions (STOPP) was used in two randomized trials involving geriatric populations, in which it contributed to a significant reduction of PIMs both at the time of discharge after acute hospitalization and up to 6 months after discharge [11], as well as reductions in the number of falls and costs [12]. Two European studies involving geriatric populations found that the number of PIMs was higher when using the STOPP criteria than when using the Beers criteria $[11,13]$. However, to our knowledge the STOPP criteria have not been previously applied to elderly haemodialysis patients.

The aims of this study were to determine the prevalence of PIMs using the STOPP criteria and the Beers criteria in elderly haemodialysis patients and to assess the association of age, sex, number of medications and co-morbidity as risk factors for PIMs in this population.

\section{Methods}

\subsection{Study Population and Data Collection}

There were 102 patients treated in the dialysis centre of Akershus University Hospital in Norway between July and December 2012, and those aged $\geq 65$ years were asked to participate in this study. Fifty-one patients were eligible for inclusion, and they all agreed to participate. The following information was collected from the electronic medical records of the patients: age, sex, number of prescribed medications, cause of kidney disease, severity of co-morbidity (classified using the Charlson Co-morbidity Index
[CCI]), time on haemodialysis and dialysis treatment quality index (quantified as urea clearance $(\mathrm{Kt} / \mathrm{V})$ ). Supplementary information about some medication side effects was collected from patient interviews, such as falls during the previous 3 months, chronic constipation (for more than 3 months before starting haemodialysis) and current dizziness when at home.

\subsection{Identification of Potentially Inappropriate Medications}

PIMs were identified using the STOPP criteria and the updated Beers Criteria [6, 14]. The STOPP criteria were developed and validated through a Delphi consensus process by 18 experts in geriatric pharmacotherapy and include 65 indicators to identify important drug-to-drug and drug-todisease interactions [6]. The STOPP criteria exhibit a high sensitivity in detecting PIMs and good inter-rater reliability $[6,15,16]$. Two physicians independently applied the STOPP and Beers criteria to review the medication lists of all of the patients included in the present study. In case of disagreement in identified PIMs between the two raters a consensus was achieved by open discussion.

We also collected information on the use of nephrologyspecific drugs, which are not included in STOPP criteria, such as phosphate binders, Vitamin D analogues, active vitamin D and erythropoietin.

\subsection{Statistical Analyses}

The data were analysed using SPSS statistical software (version 20, IBM, SPSS, Chicago, IL, USA). Descriptive statistics are presented using mean $\pm \mathrm{SD}$ or median (range) values. Fisher's exact test was used to compare the number of PIMs between groups dichotomized according to age, sex, CCI, time in dialysis and number of medications based on those equal to or above the median versus those below the median.

We applied a $5 \%$ significance cut-off in two-sided tests. Inter-rater agreement between the two raters for assessments using the STOPP and Beers screening tools is presented with \% agreement (proportion of cases for which the raters agree) and kappa coefficient for $2 \times 2$ tables. Strength of reliability coefficients was defined using the following nomenclature [17]: $<0.20$ poor, $0.21-0.40$ fair, $0.41-0.60$ moderate, $0.61-0.80$ good, $0.81-1.00$ very good.

\section{Results}

The clinical and demographic characteristics of the study population are presented in Table 1. The most common comorbidities were hypertension, cardiovascular disease and 
diabetes mellitus. The patients used a total of 652 medications and a median of 13 (range 7-21) different medications each day, and of these, a median of 5.2 (range 2- 8) drugs

Table 1 Characteristics of the study population $(n=51)$

\begin{tabular}{ll}
\hline Sex, male & $39(77)$ \\
Age, years, median (range) & $74(65-89)$ \\
Dialysis & \\
Time in haemodialysis, months, median (range) & $8(0-108)$ \\
Urea clearance, $K t / V$, mean $\pm \mathrm{SD}$ & $1.47 \pm 0.29$ \\
Arteriovenous fistula & $29(57)$ \\
Haemodialysis catheter & $22(43)$ \\
Diagnosis & \\
Nephrosclerosis & $18(35)$ \\
Post-renal disease & \\
Other & \\
Diabetic nephropathy & $8(16)$ \\
Unknown & $8(16)$ \\
Glomerulonephritis & $7(14)$ \\
Co-morbidities & $7(14)$ \\
Charlson Co-morbidity Index, median (range) & $3(6)$ \\
Hypertension & $5(2-9)$ \\
Cardiovascular disease & $47(92)$ \\
Diabetes mellitus & $28(55)$ \\
Malignancies & \\
Atrial fibrillation & $21(41)$ \\
COPD & $13(25)$ \\
Number of medications, median (range) & $12(24)$ \\
\hline Data are given as nut & $11(22)$ \\
& $13(7-21)$ \\
\hline
\end{tabular}

Data are given as number (\%) except where stated otherwise

a Post-renal disease $=$ hydronephrosis, kidney-stone disease or retroperitoneal fibrosis

${ }^{\mathrm{b}}$ Other = kidney cancer, loss of kidney graft, amyloidosis, poisoning or anti-glomerular-basement-membrane nephritis

c Malignancies = prostate cancer, colorectal cancer, testicular cancer, lung cancer, kidney cancer, lymphoma or myelomatosis

${ }^{\mathrm{d}} \mathrm{COPD}=$ chronic obstructive pulmonary disease can be defined as nephrology-specific drugs. PIMs were identified in 32 patients (63\%) by using STOPP criteria. Table 2 lists the most prevalent inappropriate medications according to the STOPP criteria. Twenty-one patients (42 \%) had one PIM and 11 patients (22\%) had two PIMs. Nineteen patients $(37 \%)$ reported falls during the previous 3 months, nine patients $(18 \%)$ reported chronic constipation before start of dialysis and three patients $(6 \%)$ reported dizziness. The most prevalent PIMs according to STOPP were proton pump inhibitors at full therapeutic dosage use ( $n=11 ; 22 \%)$, calcium-channel blockers $(n=7 ; 14 \%)$ in patients with chronic constipation and benzodiazepines $(n=6 ; 12 \%)$ in those prone to falls.

The number of PIMs did not differ significantly with age ( $\leq 74$ vs. $>74$ years, $p=0.39$ ), sex (male vs. female, $p=0.74)$, co-morbidity severity (CCI $\leq 5$ vs. $>5$, $p=1.00)$, time in haemodialysis $(\leq 8$ months vs. $>8$ months, $p=0.78$ ) or number of medications ( $\leq 13$ vs. $>13, p=0.56$ ).

Beers criteria defined PIMs in 22 patients (43\%). The most common PIM according to the Beers criteria was proton pump inhibitors (Table 3).

The kappa coefficient between raters for the STOPP criteria was $0.42(0.24-0.56,95 \% \mathrm{CI})$ and for the Beers criteria kappa was 1.0 . The \% agreement was 0.78 for STOPP and 1.00 for Beers, respectively. The disagreements occurred within two of the STOPP criteria on the interpretation of adverse effects: history of falls and chronic constipation.

\section{Discussion}

The prevalence of PIMs based on the STOPP criteria was high $(63 \%)$ in this study of elderly Norwegian haemodialysis patients. No previous studies have presented
Table 2 Potentially inappropriate medications identified by the Screening Tool of Older Persons' Prescriptions

\begin{tabular}{ll}
\hline Cardiovascular system & \\
Calcium-channel blocker with chronic constipation & $7(14)$ \\
Aspirin ( $>150 \mathrm{mg} /$ day) & $4(8)$ \\
Gastrointestinal system & $11(22)$ \\
Proton-pump inhibitor for peptic ulcer disease at full therapeutic dosage for >8 weeks & $2(4)$ \\
Loperamide & $6(12)$ \\
Central nervous system & $6(12)$ \\
Long-term administration of long-acting benzodiazepines & $2(4)$ \\
First-generation antihistamine (prolonged use for >1 week) & $2(4)$ \\
Tricyclic antidepressants with an opiate or calcium-channel blocker & \\
Opiates & $1(2)$ \\
Endocrine system & $\mathbf{4 1}$ \\
Beta-blockers in those with diabetes mellitus and frequent hypoglycaemic episodes \\
Total number of potentially inappropriate medications
\end{tabular}

Data are given as number (\%) 
Table 3 Potentially inappropriate medications identified by the Beers criteria

\begin{tabular}{l}
\hline Cardiovascular system \\
Amiodarone \\
Gastrointestinal system \\
Proton-pump inhibitors (over $>8$ weeks avoid, unless for high-risk patients, risk of Clostridium difficile infection, bone loss and \\
fractures) \\
Metoclopramide (can cause extrapyramidal effects) \\
Central nervous system \\
Selective serotonin reuptake inhibitors with a history of falls \\
Long-acting and short-acting benzodiazepines with a history of falls \\
Tricyclic antidepressants with a history of falls \\
Total number of potentially inappropriate medications \\
\hline
\end{tabular}

Data are given as number $(\%)$

use of the STOPP criteria in a haemodialysis population. However, our finding are in line with two recent studies involving patients with chronic kidney disease that applied Beers criteria $[9,10]$. In previous studies involving general geriatric populations the prevalence of PIMs ranged widely (21.4-79 \%) when applying the STOPP criteria [18-22]. The variations between the reported prevalence of PIMs can probably be attributed to differences in study populations $[6,11,13,16,20,23-25]$.

The present study chose to apply the STOPP criteria because of their documented association with a reduction in PIMs in randomized studies [11, 12], and because the STOPP criteria reportedly identify more PIMs than the Beers criteria in European elderly patients [11, 13, 23]. In the present study, the prevalence of PIMs was higher with the STOPP criteria than with the Beers criteria, which is in line with previous reports $[13,26]$. The inter-rater reliability of STOPP was lower than in previous reports [6, 15, 27]. However, these differences should be interpreted with caution because of differences in study designs and populations

Previous studies involving elderly populations found that the number of PIMs was positively associated with the number of medications, age and number of co-morbidities $[11,23,25]$. In contrast, the present study found no associations between the number of PIMs and age, sex, comorbidity severity, time on haemodialysis and number of medications. This difference between the studies may be due to differences in the case mix between the populations or the small number of participants in the present study.

This study had several limitations. The patients were relatively old and the number of patients was small, and so the results cannot be generalized to all patients with ESRD. The small sample size also limited the analysis of risk factors for PIMs, and multivariable analysis was not considered meaningful. The study did not include a comparison group. Finally, the STOPP criteria were not developed specifically for use in patients with ESRD and they do not evaluate several drugs commonly used by patients with renal impairment, such as phosphate binders, erythropoietin, potassium binders and calcimimetics.

\section{Conclusions}

In conclusion, the prevalence of PIMs among elderly haemodialysis patients was high when applying the STOPP criteria and the Beers criteria, even though these criteria do not evaluate nephrology-specific drugs. In contrast, the STOPP criteria identified more PIMs compared with the Beers criteria in our study population. This finding supports the use of medication review to identify and avoid PIMs in haemodialysis patients, who are typically exposed to polypharmacy.

\section{Compliance with Ethical Standards}

Ethical Approval This study was approved by the ethics committee of Akershus University Hospital and was performed in accordance with the ethical standards of the Declaration of Helsinki. All participants gave their consent at the start of the study.

Funding No funding was received for the conduct of the study or the preparation of this manuscript.

Conflict of interest Krystina Parker, Willy Aasebø and Knut Stavem have no conflicts of interest.

Open Access This article is distributed under the terms of the Creative Commons Attribution-NonCommercial 4.0 International License (http://creativecommons.org/licenses/by-nc/4.0/), which permits any noncommercial use, distribution, and reproduction in any medium, provided you give appropriate credit to the original author(s) and the source, provide a link to the Creative Commons license, and indicate if changes were made.

\section{References}

1. KDIGO. Kidney disease improving global outcome. 2013. http:// kdigo.org/home/guidelines/. 
2. KKDOQI. 2015. https://www.kidney.org/professionals/guidelines/ hemodialysis 2015.

3. Kaur S, Mitchell G, Vitetta L, et al. Interventions that can reduce inappropriate prescribing in the elderly: a systematic review. Drugs Aging. 2009;26(12):1013-28.

4. Lutters M, Harbarth S, Janssens JP, et al. Effect of a comprehensive, multidisciplinary, educational program on the use of antibiotics in a geriatric university hospital. J Am Geriatr Soc. 2004;52(1):112-6.

5. St Peter WL. Management of polypharmacy in dialysis patients. Semin Dial. 2015;28(4):427-32.

6. Gallagher P, Ryan C, Byrne S, et al. STOPP (Screening Tool of Older Person's Prescriptions) and START (Screening Tool to Alert doctors to Right Treatment). Consensus validation. Int J Clin Pharmacol Ther. 2008;46(2):72-83.

7. Beers MH. Explicit criteria for determining potentially inappropriate medication use by the elderly. An update. Arch Intern Med. 1997;157(14):1531-6.

8. McLeod PJ, Huang AR, Tamblyn RM, et al. Defining inappropriate practices in prescribing for elderly people: a national consensus panel. CMAJ. 1997;156(3):385-91.

9. Kondo N, Nakamura F, Yamazaki S, et al. Prescription of potentially inappropriate medications to elderly hemodialysis patients: prevalence and predictors. Nephrol Dial Transplant. 2015;30(3):498-505.

10. Jones SA, Bhandari S. The prevalence of potentially inappropriate medication prescribing in elderly patients with chronic kidney disease. Postgrad Med J. 2013;89(1051):247-50.

11. Gallagher PF, O'Connor MN, O'Mahony D. Prevention of potentially inappropriate prescribing for elderly patients: a randomized controlled trial using STOPP/START criteria. Clin Pharmacol Ther. 2011;89(6):845-54.

12. Frankenthal D, Lerman Y, Kalendaryev E, et al. Intervention with the screening tool of older persons potentially inappropriate prescriptions/screening tool to alert doctors to right treatment criteria in elderly residents of a chronic geriatric facility: a randomized clinical trial. J Am Geriatr Soc. 2014;62(9):1658-65.

13. Hamilton $\mathrm{H}$, Gallagher $\mathrm{P}$, Ryan $\mathrm{C}$, et al. Potentially inappropriate medications defined by STOPP criteria and the risk of adverse drug events in older hospitalized patients. Arch Intern Med. 2011;171(11):1013-9.

14. American Geriatrics Society 2015 updated Beers criteria for potentially inappropriate medication use in older adults. J Am Geriatr Soc. 2015;63(11):2227-46.
15. Gallagher P, Baeyens JP, Topinkova E, et al. Inter-rater reliability of STOPP (Screening Tool of Older Persons' Prescriptions) and START (Screening Tool to Alert doctors to Right Treatment) criteria amongst physicians in six European countries. Age Ageing. 2009;38(5):603-6.

16. Ryan C, O'Mahony D, O'Donovan DO, et al. A comparison of the application of STOPP/START to patients' drug lists with and without clinical information. Int J Clin Pharm. 2013;35(2):230-5.

17. Altman DG. Practical statistics for medical research. London: Chapman \& Hall; 1991.

18. Garcia-Gollarte F, Baleriola-Julvez J, Ferrero-Lopez I, et al. Inappropriate drug prescription at nursing home admission. J Am Med Dir Assoc. 2012;13(1):83.e9-15.

19. Ryan C, O'Mahony D, Kennedy J, et al. Potentially inappropriate prescribing in an Irish elderly population in primary care. Br J Clin Pharmacol. 2009;68(6):936-47.

20. Hill-Taylor B, Sketris I, Hayden J, et al. Application of the STOPP/START criteria: a systematic review of the prevalence of potentially inappropriate prescribing in older adults, and evidence of clinical, humanistic and economic impact. J Clin Pharm Ther. 2013;38(5):360-72.

21. Liu CL, Peng LN, Chen YT, et al. Potentially inappropriate prescribing (IP) for elderly medical inpatients in Taiwan: a hospital-based study. Arch Gerontol Geriatr. 2012;55(1):148-51.

22. Parsons C, Johnston S, Mathie E, et al. Potentially inappropriate prescribing in older people with dementia in care homes: a retrospective analysis. Drugs Aging. 2012;29(2):143-55.

23. O'Sullivan DP, O'Mahony D, Parsons C, et al. A prevalence study of potentially inappropriate prescribing in Irish long-term care residents. Drugs Aging. 2013;30(1):39-49.

24. Frankenthal D, Lerman Y, Lerman Y. The impact of hospitalization on potentially inappropriate prescribing in an acute medical geriatric division. Int J Clin Pharm. 2015;37(1):60-7.

25. Onatade R, Auyeung V, Scutt G, et al. Potentially inappropriate prescribing in patients on admission and discharge from an older peoples' unit of an acute UK hospital. Drugs Aging. 2013;30(9):729-37.

26. Gallagher P, Lang PO, Cherubini A, et al. Prevalence of potentially inappropriate prescribing in an acutely ill population of older patients admitted to six European hospitals. Eur J Clin Pharmacol. 2011;67(11):1175-88.

27. Ryan C, O'Mahony D, Byrne S. Application of STOPP and START criteria: interrater reliability among pharmacists. Ann Pharmacother. 2009;43(7):1239-44. 